\title{
Ex-nihilo: Obstacles Surrounding Teaching the Standard Model
}

\author{
Kevin A. Pimbblet \\ Address: Department of Physics, University of Durham, South Road, Durham, DH1 3LE, UK. \\ E-mail: K.A.Pimbblet@durham.ac.uk
}

\begin{abstract}
The model of the Big Bang is an integral part of the national curriculum for England. Previous work (e.g. Baxter 1989) has shown that pupils often come into education with many and varied prior misconceptions emanating from both internal and external sources. Whilst virtually all of these misconceptions can be remedied, there will remain (by its very nature) the obstacle of ex-nihilo, as characterised by the question 'how do you get something from nothing?' There are two origins of this obstacle: conceptual (i.e. knowledge-based) and cultural (e.g. deeply held religious viewpoints). The article shows how the citizenship section of the national curriculum, coming 'online' in England from September 2002, presents a new opportunity for exploiting these.
\end{abstract}

Keywords: High School, Astronomy/Cosmology, Social/Cultural

\section{Introduction}

The model of the Big Bang (The Standard Model herein; e.g. Peebles 1993) at Key Stage 4 is a requisite part of science and physics teaching in England (i.e. contained within the framework of the National Curriculum; 3d: 'about some ideas used to explain the origin and evolution of the Universe'). It is common for trainee teachers to be given background information (typically based upon lectures courses and planetarium visits) on the teaching of astrophysics and cosmology.

Even if a teacher is a consummate professional who has been trained in the teaching of astrophysics, however, one finds that there is a miasma of obstacles in the way of successfully teaching the Standard Model. Indeed, Comins (1993) prepares a list of over 550 individual misconceptions related to the teaching of astrophysics. These misconceptions fall roughly into three camps: those caused by incorrect internal (mental) processes, those caused by incorrect external processes (inaccurate information from teachers, parents and peers), and those caused by a combination of both internal and external factors. Whilst there exists plenty of evidence in the literature for the causes 
of such misconceptions, there is little in the way of strategies to deal with them.

\section{Obstacles}

Research by a number of different authors (e.g. Baxter 1989; Sneider \& Pulos 1983; Nussbaum, 1979) suggests that children and juveniles will start to formulate their own ideas about the natural world long before any formal education is given. Inevitably, such self generated formulations will be riddled with both internal and external misconceptions. As their formal education progresses, these scientific misconceptions should be systematically dealt with during the course of time. For example, Baxter (1989) cites the case of a pupil persuaded towards an Aristotelian point of view.

Such misconceptions can readily be corrected. To this end, Bennett (2000) advocates the use of a five step plan based upon the un-learning of these misconceptions. By establishing a contextual framework, Bennett (2000) argues that misconceptions about astronomy can expediently be corrected. Consider the case of a pupil harbouring an Aristotelian viewpoint. Aristotle reasoned that the Earth was stationary in space and the Sun, Moon, other planets and stars orbit around it in circles. Later, Ptolomy refined this view with his theory of celestial spheres: the Earth was at the centre of the Universe and each extra-terrestrial body moved its own sphere with the outermost sphere consisting of all of the (fixed) stars. Knowing that this left room for Heaven and Hell outside of these spheres, the church adopted this theory. Observations consistently disagreed with this view, the primary one being retrograde (backwards) movement of planets across the sky. Although attempts were made to understand this movement (e.g. Ptolomy proposed that planets may execute epicycles) it was not until the seventeenth century that Galileo, having learnt of the invention of and subsequently built his own telescope, provided conclusive observations against the geocentric model of Aristotle. He showed that the satellites of Jupiter (later known as the Galilean satellites) do not orbit Earth but orbit Jupiter instead. Earth need not be at the centre of everything. Indeed, Galileo showed too that Venus exhibits phases, which can be explained if both Venus and the Earth orbit the Sun. Further, Kepler's development from the heliocentric model of Copernicus demonstrated elegantly how retrograde motion could be explained in a simple manner. Further problematic observations for the geocentric model in the seventeenth century included Galileo demonstrating that sunspots were not shadows of planets passing in front of the Sun, rather actual spots on its surface that rotated; and the supernova of 1604 whose parallax demonstrated that it was located beyond the planets and thus the heavens were subject to change. Presentation 
of these observational arguments would readily challenge and correct an Aristotelian viewpoint.

There is, however, only so far that this technique can be applied before a terminal problem arises. This obstacle can be characterised by the question 'how do you get something from nothing?' (the ex-nihilo obstacle, if you will). This question can be divided into two aspects: a conceptual problem and a cultural problem.

Scientific understanding and knowledge of how to tackle the ex-nihilo obstacle is, perhaps, at a level above and beyond what an average physics teacher is capable of addressing. Indeed, it is probable that said teacher has had little theoretical grounding in cosmology and the Standard Model; depending, of course, where they were trained and what courses they undertook as an undergraduate (Baxter 1991; Baxter et al. 1991). Even if they do possess such knowledge, answering the characteristic question given above would require great expertise and teaching to effectively give an answer appropriate at Key Stage 4. The crucial problem is that the theory of general relativity (e.g. Einstein 1950) requires modification when density tends to infinity (as happens when we reverse time towards the Big Bang; Peebles 1993) to take account of quantum effects. Since we do not possess a complete theory of quantum gravity, we can presume that our theories are only valid for times after quantum effects became small. This limit of general relativity is the Planck time, about $10^{-43} s$ after the Big Bang. We can only make extrapolations to the Planckian era based upon theory and conjecture, which are typically only accessible at the post-doctoral level.

Inevitably, by the very nature of cosmology, the answering of such a characteristic question is likely to come into conflict with pupils who possess strongly held religious beliefs (e.g. creationism). This conflict cannot be simply resolved by citing scientific evidence: these pupils' views amount to belief, and are based on faith.

\section{Discussion}

In attempting to answer the ex-nihilo obstacle, there are several tactics that a teacher may employ. To confront the conceptual problem requires an acknowledgement that scientific understanding and knowledge is still being developed to this day. For example, since the Hubble Space Telescope (HST) was launched 1990 it has provided astronomers with a plethora of new data to characterize and explain. Indeed, one of the HST key projects were observations of distant supernovae to determine how the Universe is expanding. This led to the startling discovery that the Universe is not only expanding, but accelerating (e.g. Perlmutter et al. 1999). These observations are forcing 
astronomers to reconsider their cosmological models. We can only guess at what evidence the next generation space telescope may reveal. Science is, after all, characterised by evidence-based argument. Accordingly, there is no one theory that describes everything in the Universe without flaw.

Teachers are therefore advised to make appropriate citations to evidence for the Big Bang. Starting simply, the expansion of the Universe explains why light captured from distant galaxies displays similar line spectra to light in a laboratory but is shifted to longer wavelengths (e.g. Hubble \& Humason 1931). At a more sophisticated level, the cosmological microwave background radiation (CMBR; Penzias \& Wilson 1965) provides more evidence for the Big Bang.

If the Universe came from a previous epoch that was highly compressed and hot, the radiation from that epoch must still be with us today. When the temperature of the Universe was billions of Kelvin, such radiation would have been in the form of $\gamma$-rays. As the Universe expanded, however, it cooled and the radiation's wavelength increased (recall Wein's law). Gamow and others calculated that this radiation at the present epoch would be in the microwave part of the electromagnetic spectrum (e.g. if the temperature is about $5 \mathrm{~K}$, then the wavelength of the radiation will be $\lambda \sim$ $0.06 \mathrm{~cm})$. Therefore, interpreted as highly redshifted radiation from a primeval fireball, the ubiquity of the CMBR gives excellent evidence of a previous state of the Universe that was extremely hot. Both the redshifted Universe and the CMBR can be explained at Key Stage 4 level. Whilst the Big Bang model explains these and many other observations, historically it has not been without criticism. For example, one may enquire, why is the Universe so close to the critical density? For the moment, scientists accept the Big Bang model as it ties together many threads of evidence. Yet, they must also be sceptical: the model has had problems in the past and will no doubt have problems in the future too.

The cultural problem is a much thornier issue. Contained within the declaration of human rights is the right to freedom of religion. The teacher, being in a responsible role, must ensure that they do not force their own (scientific) viewpoints upon such a pupil. Rather, they must allow the pupil to come to their own conclusions after presentation of the scientific evidence that supports the Standard Model. To force a viewpoint upon a pupil would be more than counter-productive. Such force could generate resentment towards the teacher, and lead to rejection of the subject material without consideration.

Since these dichotic opinions potentially lead to conflict, I advocate the use of a full classroom discussion to debate the issues surrounding the ex-nihilo obstacle. A discussion provides excellent 
opportunities for cross-curricular activity whilst allowing the pupils to express their own opinions. Research skills (to back up any scientific argument; for example, see Stephen Hawking's Universe series at www.pbs.org/wnet/hawking), information technology skills (to present ideas with) and literacy can all play a significant role in such a discussion. Other subject areas could readily be drawn upon as well (e.g. drama).

More significantly, with the subject of citizenship coming 'online' in England from September 2002, an already squashed timetable could readily accommodate aspects of citizenship within an astronomical origins debate. Particular items in the citizenship national curriculum programme of study include (at Key Stage 4) 1b ('the origins and implications of the diverse national, regional, religious and ethnic identities in the United Kingdom and the need for mutual respect and understanding'); 2 (all points under 'developing skills of enquiry and communication' relevant). Not only will such a discussion enhance pupil development, it would also actively promote citizenship though astronomy. It must be emphasized, however, that pupils need to be carefully guided to preserve mutual respect.

\section{Conclusions}

This work has reviewed some research on the misconceptions encountered during the teaching of astrophysics. When corrected, these misconceptions will generally be dealt with effectively, but there will often remain an ex-nihilo obstacle.

Such an obstacle can be divided into two separate issues: a conceptual (knowledge-based) one and a cultural one. In tackling these issues, one must address:

1. Science is still developing to this day and we do not presently possess a theory that describes everything without flaw.

2. Some pupils will hold religious values that may appear contradictory to the Standard Model.

Since both of these points lead to a potential conflict and debate, a full discussion is therefore advocated, simultaneously developing aspects of the national curriculum for citizenship. 


\section{Acknowledgements}

The preparation of this manuscript has made use of resources at both the University of Durham and

St.Leonard's R.C.V.A. School, Durham. KAP thanks Mary C. Hawkrigg for her reading through of the text and for providing useful suggestions that have improved this work. Finally, thanks to John Newman, Marion E. Jones, Peter Little and Simon B. Campbell for most unknowingly inspiring this work and for providing copious amounts of feedback on my teaching.

\section{References}

Baxter, J., 1989, International Journal of Science Education, 11, 502

Baxter, J., 1991, Quart. Journal of the Royal Astron. Soc., 32, 147

Baxter, J., et al., 1991, Quart. Journal of the Royal Astron. Soc., 32, 159

Bennett, J., 2000, American Astron. Soc. Meeting, 197, 86.01

Comins, N.F., 1993, Bulletin of the American Astron. Soc., 25, 1430

Einstein, A., 1950, 'The Principle of Relativity', Metheun, London

Hubble, E. \& Humason, M. L. 1931, Astrophysical Journal, 74, 43

Nussbaum, J., 1979, Science Education, 63, 83

Peebles, P.J.E., 1993, 'Principals of Physical Cosmology', Princeton Series in Physics, Princeton University Press, NJ

Penzias, A. A. \& Wilson, R. W. 1965, Astrophysical Journal, 142, 419

Perlmutter, S. et al., 1999, Astrophysical Journal, 517, 565

Sneider, C. \& Pulos, S., 1983, Science Education, 67, 205 